\title{
Laughter Detection in the Wild: Demonstrating a Tool for Mobile Social Signal Processing and Visualization
}

\author{
Simon Flutura, Johannes Wagner, Florian Lingenfelser, Andreas Seiderer, Elisabeth André \\ Human Centered Multimedia, Augsburg University, Augsburg, Germany
}

\{lastname\}@hcm-lab.de

\begin{abstract}
In this demo, we present MobileSSI, a flexible software framework for Android and embedded Linux platforms, that provides developers with tools to record, analyze and recognize human behavior in real-time on mobile devices. To illustrate the benefits of the framework for the analysis of social group dynamics in naturalistic mobile settings, we present a demonstrator for laughter recognition that was implemented with MobileSSI. The demonstrator makes use of smartphones for sensing and analyzing data and employs smartwatches and tablets for visualizing the results and providing user feedback. To enable communication within the resulting ecology of mobile devices, MobileSSI includes a web socket plugin.
\end{abstract}

\section{CCS Concepts}

$\bullet$ Human-centered computing $\rightarrow$ Open source software; Ubiquitous and mobile computing systems and tools;

\section{Keywords}

Social Signal Processing; Affective Computing; "In the Wild" Studies

\section{INTRODUCTION}

While there is a proliferation of studies that investigate specific aspects social human-human communication under laboratory-like conditions, hardly any attention has been paid to naturalistic social settings in which humans interact with each other. It is easy to understand that researchers have a preference for controlled settings. Nevertheless, it is disappointing to see that many problems have been satisfactorily solved under static laboratory conditions, but still fail when applied in a "real world" setting. To foster the development of social signal processing applications in the wild, we decided to develop MobileSSI, a port of the Social Signal Interpretation (SSI) framework 4 to Android and embedded Linux platforms. The framework supports the

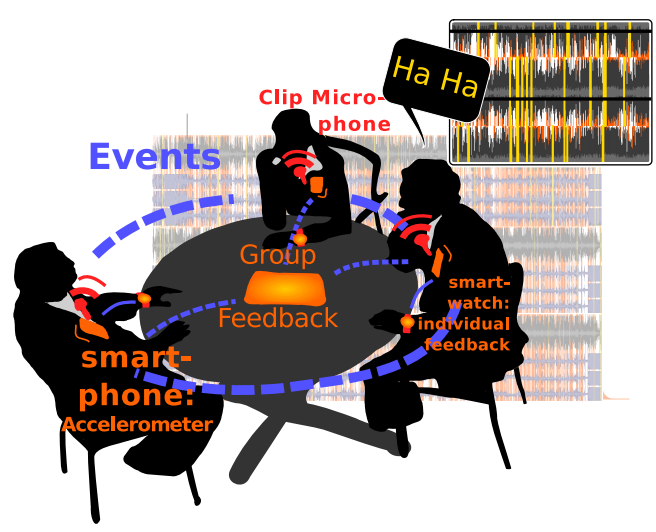

Figure 1: Setting of the laughter demonstrator.

joint development of processing pipelines for the analysis of social signals on a desktop computer and mobile devices. To illustrate the practical use of the framework, we will present a demonstrator implemented on the basis of MobileSSI that recognizes laughter in naturalistic social settings (see Figure 1). Laughter is an important indicator of enjoyment in social groups [3]. Furthermore, it has a positive effect on people's wellbeing 1]. Consequently, techniques for real-time laughter recognition enable a wide range of applications including social coaching and health care systems. Conferences, such as ICMI, are places where people meet and interact spontaneously and of course also engage in laughter. We therefore believe that ICMI provides a good setting to showcase our demonstrator "in the wild".

\section{THE FRAMEWORK}

MobileSSI provides tools to filter, transform, classify and fuse data streams in real-time and to display information, locally on mobile devices. To integrate data from multiple sensors, it relies on an asynchronous vector-based fusion approach which also enables us to cope with missing and noisy data 3. It comes with a WebSocket plugin that supports communication within an ecology of mobile devices including smartphones, smartwatches and tablets. MobileSSI adopts the core idea of SSI to accomplish complex signal processing pipelines from simple reusable units. The structure of a pipeline is described using plain XML which enables us to define a pipeline independent of the platform it will run on. Nevertheless, to conduct social signal processing in the wild, a number of challenges have to be met. 


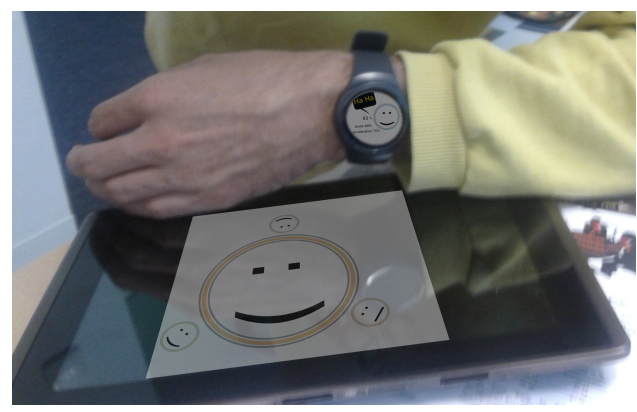

Figure 2: Devices used for information visualization in the laughter demonstrator

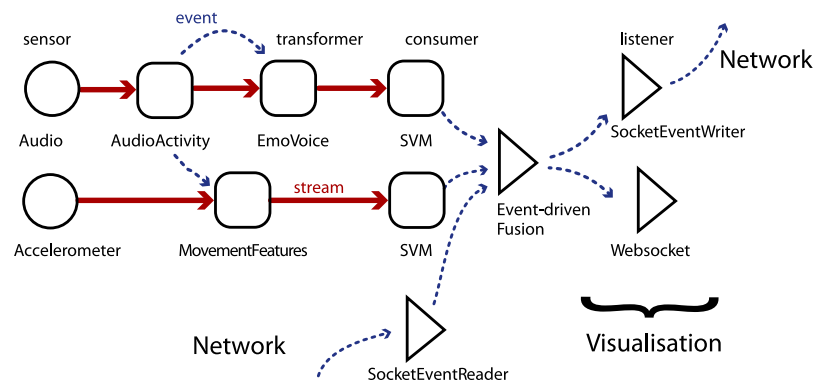

Figure 3: Sketch of the recognition pipeline (signal flow from left to right): features are extracted from the raw streams when voice activity is detected in the audio channel. Support Vector Machine (SVM) classifiers recognize the presence of laughter in the channels. Laughter events from both modalities are fused with events received over the network and visualized through the websocket interface.

For example, for the laughter detector, we had to rely solely on mobile sensor technology and therefore decided to stick to audio and accelerometer sensors.

Figure 3 shows the components of a pipeline that has been created for the laughter demonstrator. It includes mobile audio and accelerometer sensors, transformers applying features on the captured signals as well as consumers for classification and output. To orchestrate a multitude of mobile devices, the following components have been added to the pipeline:

- SocketEventWriter: sends events over network

- SocketEventReader: receives events to fuse them in the recognition pipeline

- Websocket: provides an HTTP and WebSocket server used to host a web page on the device that can be displayed on smartwatches and the tablet

\section{DEMO SETUP}

In our laughter demonstrator, we combine smartphones as sensing devices with smartwatches as personal and tablets as group displays. While users engage in social interactions with each other, MobileSSI records and synchronizes data from the audio and accelerometer sensors embedded in the mobile smartphones worn in the users' breast pocket. Using an event-driven fusion approach, the users' audio and accelerometer data are integrated to determine their degree of enjoyment. Smartwatches and tablets display information on aggregated cues of enjoyment at the individual and the group level in real-time (see Figure 4 .
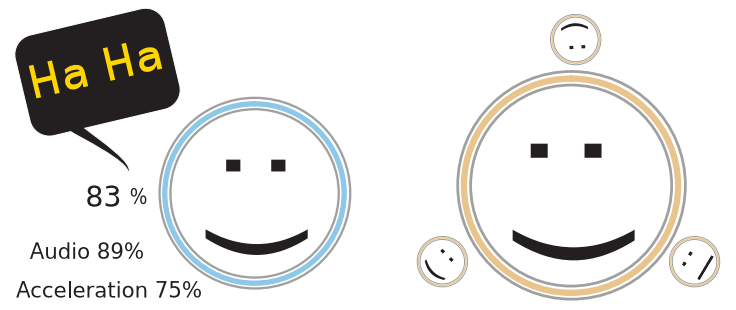

Figure 4: Visualization of enjoyment at the individual (left) and the group level (right)

\section{CONCLUSION}

This paper presents a novel framework called MobileSSI for recording and analyzing social signals in the wild. To validate the framework, we implemented an application for real-time laughter recognition that integrates the data from audio and accelerometer sensors embedded in smartphones and displays aggregated information on various mobile displays, such as smartwatches or tablets. The demo will demonstrate (i) the specification of pipelines for fusing mobile data and (ii) the execution of the pipelines to detect social cues in mobile settings. At the example of laughter recognition, we will show the capability of MobileSSI to run complex signal processing and machine learning tasks locally on mobile devices. However, MobileSSI is not limited to this kind of application, but offers a flexible software framework to foster the development of a wide range of applications that conduct social signal processing in the wild. MobileSSI is open source and available for the publi ${ }^{1}$ A more detailed description can be found in our regular ICMI paper [2].

\section{REFERENCES}

[1] L. S. Berk, S. A. Tan, W. F. Fry, B. J. Napier, J. W. Lee, R. W. Hubbard, J. E. Lewis, and W. C. Eby. Neuroendocrine and stress hormone changes during mirthful laughter. The American journal of the medical sciences, 298(6):390-396, 1989.

[2] S. Flutura, J. Wagner, F. Lingenfelser, A. Seiderer, and E. André. MobileSSI - Asynchronous Fusion for Social Signal Interpretation in the Wild. In Proceedings of the 18th International Conference on Multimodal Interaction, 2016.

[3] F. Lingenfelser, J. Wagner, E. André, G. McKeown, and W. Curran. An event driven fusion approach for enjoyment recognition in real-time. In Proceedings of ACM Multimedia, pages 377-386, 2014.

[4] J. Wagner, F. Lingenfelser, T. Baur, I. Damian, F. Kistler, and E. André. The Social Signal Interpretation (SSI) framework: multimodal signal processing and recognition in real-time. In Proceedings of ACM Multimedia, pages 831-834, 2013.

${ }^{1}$ https://hcmlab.github.io/mobileSSI/ 